\title{
Is Gradual and Controlled Approach to Herd Protection a Valid Strategy to Curb the COVID-19 Pandemic?
}

\author{
RAMANAN LAXMINARAYAN ${ }^{*}$ AND T JACOB JOHN ${ }^{2}$ \\ ${ }^{1}$ Centre for Disease Dynamics, Economics \& Policy, Washington DC andSenior Research Scholar, Princeton University, United \\ States; and ${ }^{2}$ Retired Professor, Department of Clinical Virology, Christian Medical College, Vellore, India. *ramanan@cddep.org
}

Published online: May 06, 2020; PII: S097475591600175

$\mathrm{P}$ andemics have their own natural history. They are the sum of epidemics in many countries. Most pandemics in the twentieth and twentyfirst centuries have been caused by viruses influenza, chikungunya, HIV/AIDS and now the coronavirus disease (COVID-19), caused by the severe acute respiratory syndrome coronavirus 2 (SARS-CoV-2). When the virus is transmitted by the respiratory route, it goes around the world in a few months - pandemic flu H1N1 of 2009 was world-wide in about 3 months; COVID19 in 4 months.

The risk to children is reported to be small [1], but in India, with 130 million children under the age of five, the numbers with severe disease and death can add up to large numbers during the epidemic period.Additionally, with many undernourished children and risk-factors like environmental and household air pollution, the epidemic can be quite worrisome for pediatricians.

Pediatric patients reportedly acquire COVID-19 either through close contact with infected family members $(89 \%)$, exposure to endemic areas (33\%), or both $(22 \%)$; with the majority (53\%) showing moderate symptoms and no severe or critical cases [2]. Though symptoms of COVID-19 are often mild in children, the prevalence of pneumonia with COVID-19 (53\%) in this study is higher than with H1N1 influenza (11\%), and similar to SARS $(65 \%)$ [2]. This again points to the impendingchallengesin dealing with COVID-19 in children.

As pediatricians look forward to the future beyond the current extended lockdown, an important question relates to how we can navigate out of the current crisis while incurring minimal casualties, primarily among the elderly but also in the pediatric population. Globally, there are two broad approaches to taming the epidemic: by imposing lockdowns and other forced physical distancing measures to 'flatten the curve',or allowing 'herd immunity' by allowing for a graded acquisition of immunity.
We do not endorse the idea of letting the epidemic a free hand in order to create sufficient herd immunity to end the epidemic;as it would entail an enormous burden on the healthcare system - United Kingdom, at first, considered a different approach - of unrestricted spread of disease without any brakes applied, but public health experts were able to convince the government to accept the more reasonable mitigation approach. We should clarify at the outset that an approach of uncontrolled spread, one that no country is following at this time, would be a terrible mistake.

In general, a combination of mitigation and controlled herd immunity is the intervention adopted by most, if not all countries. Lockdown, cough/sneeze etiquette, hand washing (with soap and water) every time any surface potentially contaminated by droplets is touched, wearing masks in human presence, and physical distancing are the current interventions for risk-mitigation towards flattening the curve. The current approach in India appears to be based on the idea that the disease can be contained and eventually eliminated through risk mitigation plus tracing potentially infected persons through contact-tracing, testing and quarantining. In the unlikely event of this seemingly impossible task were to be accomplished, the country will have to be in a perpetual state of alert for screening and quarantining anyone who might be infected and arriving in the country. As we have observed in the past, asymptomatic infected can slip past border screenings and introduce SARSCOV-2 into the general population.

The epidemic will decline when herd immunity reaches sufficient level, determined by calculation based on the basic reproduction number, Ro. The reproductive number is the number of secondary infections produced by a single primary infection in a completely naïve population. The higher the Ro, the greater is the herd immunity that is required to prevent outbreaks or to 
eliminate infection from the nation. For COVID-19, the Ro has been reported to be $\sim 2$ in early studies [3]. Recently US CDC has described Ro of 5.7 - we think that the latter pertains to coronavirus infection, the majority of which are asymptomatic. The former applies to COVID-19. The epidemiological estimate is that we need $70 \%$ herd immunity to turn the epidemic down for a Ro of 2 and $80 \%$ herd immunity if the Ro was 5.7. The proportion of the population that should be exposed to the virus for herd immunity to be effective is calculated as 1-1/Ro.

In the absence of serological studies, the true extent of spread of SARS-COV-2 in India is unknown. However, evidence from Wuhan, and more recently from France suggests that the number of asymptomatic patients may be as much as four times the number of patients who show symptoms $[4,5]$. At the time of writing, India has nearly 50,000 reported cases and 1700 reported deaths. Assuming an undercount by a factor of three and then adjusting for asymptomatic patients, it is likely that there are over 500,000 infections in the country.

Our view is that given the large number of asymptomatic patients, the utility of case identification and containment is fast diminishing and our best bet may lie in cluster containment. With these measures also, we can only slow down the epidemic and not stop transmission entirely. We then have only the option of holding down the epidemic through periodic shutdowns until a vaccine arrives. However, it is entirely possible that herd protection, consequent to the build-up of sufficient level of herd immunity may arrive before a vaccine does. In other words, if about $65-70 \%$ of our population is infected and acquires immunity, it becomes less likely that an infected individual comes into contact with a person susceptible to infection. At the tipping point, each infected person, on average, infects less than one other person; and here onwards, the number of cases will decline.

Furthermore, there are still many unknowns. The degree of protection afforded by antibodies among exposed is still unclear. There is evidence of immune response from the fact that patients with COVID-19 have cleared the infection. The result could either be sterilizing immunity, where re-infection can be ruled out, or weak immunity that wanes over time, and at best dampens symptoms in case of re-infection. We are not sure which end of the spectrum recovered patients from COVID-19 would fall into. It is also not known if those that have had mild or asymptomatic infections have the same degree of immunity and protection as those with symptomatic infections. A recent study showed that even asymptomatic patients had high viral loads approaching those of symptomatic patients [6].
Evidence from people who survived SARS and MERS suggests that antibodies persist for two years for SARS [7], while antibodies to MERS last nearly three years [8]. Early evidence from SAR-COV-2 from the Netherlands indicates that most PCR-confirmed SARS-CoV-2infected persons had seroconverted by 2 weeks after disease onset [9]. All of this indicates that there is a strong likelihood of some protection through exposure to the virus and even subclinical infection.

Initial estimates from various sources prior to the lockdown were that roughly $25 \%$ of the populationwould be infected during the first wave of infection [10]. While the projected number of infections has declined significantly, because of the lockdown associated reduction in transmission, the spread of the virus will pick up speed when the lockdowns are removed. At this stage, early identification of high-risk groups, including both children and the elderly would be important to avert severe infections.

From that point on, the dual strategy of slowing spread while protecting vulnerable populations should be our main options to reach the level of herd protection that will ensure that the entire population is protected until such time that we have vaccination options. However, the likely situation post-pandemic will be endemic transmission, possibly with seasonal low and high incidences - low in summer but high during monsoons or winter.

To conclude, gradual and staged acquisition of herd protection could be a perfectly legitimate public health goal. As with all public health decisions made at a time of uncertainty, there are downside risks and there are risks and costs associated with alternative approaches as well. Available control measures, including lockdowns, should be applied selectively with the understanding that this may be inevitable and perhaps even desirable, as long as the ability of the health system to handle hospitalizations is not overwhelmed.

Disclaimer: This article represents the personal opinions of the authors and does not reflect the institutional opinion of their employers.

Funding: None; Competing interests: None stated.

\section{REFERENCES}

1. Jordan RE, Adab P, Cheng KK. Covid-19: Risk factors for severe disease and death. BMJ.2020;368:m1 198. Available from:https://www.bmj.com/content/368/bmj.m1198.long. Accessed April 17, 2020.

2. Qiu H, Wu J, Hong L, Luo Y, Song Q, Chen D. Clinical and epidemiological features of 36 children with coronavirus disease 2019 (COVID-19) in Zhejiang, China: An observational cohort study.Lancet Infect Dis. 2020 Mar 25. Available from: https://www.thelancet. com/journals/laninf/ 
article/PIIS1473-3099(20)30198-5/fulltext.Accessed April 17, 2020.

3. Sanche S, Lin YT, Xu C, Romero-Severson E, Hengartner N, $\mathrm{Ke}$ R. High contagiousness and rapid spread of severe acute respiratory syndrome coronavirus 2. Emerg Infect Dis. 2020. Available from:https://wwwnc.cdc.gov/eid/article/26/ 7/20-0282_article. Accessed April 17, 2020.

4. Salje H, Kiem CT, Lefrancq N, Courtejoie N, Bosetti P, PaireauJ, et al. Estimating the burden of SARS-CoV-2 in France.2020. ffpasteur-02548181f. Available from:https:// hal-pasteur.archives-ouvertes.fr/pasteur-02548181/ document. Accessed April 17, 2020.

5. Li R, Pei S, Chen B, Song Y, Zhang T, Yang W, Shaman et al. Substantial undocumented infection facilitates the rapid dissemination of novel coronavirus (SARS-CoV2). Science. 2020:368:489-93.

6. Zou L, Ruan F, Huang M, Liang L, Huang H, Hong Z, et al. SARS-CoV-2 viral load in upper respiratory specimens of infected patients. New Engl J Med.2020;382:1177-9.
7. Mo H, Zeng G, Ren $\mathrm{X}$, Li H, Ke C, Tan $\mathrm{Y}$, et al. Longitudinal profile of antibodies against SARScoronavirus in SARS patients and their clinical significance.Respirology. 2006;11:49-53.

8. Payne DC, Iblan I, Rha B, Alqasrawi S, Haddadin A, Al Nsour M, et al. Persistence of antibodies against MiddleEast respiratory syndrome coronavirus. Emerg Infect Dis. 2020;22:1824-6.

9. Okba NMA, Müller MA, Li W, Wang C, vanKessel CHG, Corman VM, et al. Severe acute respiratory syndrome coronavirus 2 specific antibody responses in coronavirus disease 2019 patients.Emerg Infect Dis. 2020 Apr 8. Available from:https://wwwnc.cdc.gov/eid/article/26/7/200841_article. Accessed April 17, 2020.

10. Mandal S, Bhatnagar T, Arinaminpathy N, Agarwal A, Chowdhury A, Murhekar M, et al. Prudent public health intervention strategies to control the coronavirus disease 2019 transmission in India: A mathematical model-based approach. Indian J Med Res. 2020;151:190-9. 